\title{
Determination of Erosivity of Enugu State
}

\author{
G.I. Okolotu, S.I. Oluka, P.C. Eze.
}

Department of Agricultural and Bioresource Engineering, Enugu State University of Science and Technology, Enugu, Nigeria.

\begin{abstract}
To combat erosion, there is need for adequate examination of soils and factors of erosion. The study of Erosivity is vital for effective soil conservational planning and agricultural activities in Enugu State of Nigeria of West Africa, and other parts of the world. In this study, rainfall amount and duration were obtained and used in the determination of rainfall intensities in Enugu regions. These rainfall records were used in calculating the three erosivity indices namely; the maximum 30-minute intensity, kinetic energy greater than 25, and peak storm intensity. With the aid of the above data, erosivity of Enugu was uncovered. This was achieved by taking the average of the calculated years. This Erosivity value can be used in modeling a general soil loss equation for all soil types in Enugu. This general soil loss equation can be used to predict erosion in Enugu region. Also, from the force impact of rain capable of causing erosion, erosive rain were separated from non erosive rain using the method based upon the concept that there is a threshold value of intensity at which rain starts to be erosive. With this insight, one can also predict future erosive rain and non erosive rain using the weather forecast data. With the knowledge of these predictions, erosion prevention technique may be applied in areas of possible future occurrence rather than remediating soil after erosion hazard.
\end{abstract}

Keywords - Erosivity, Soil Erosion, Rainfall, Soil Loss, Storm Intensity.

\section{INTRODUCTION}

Erosivity refers to the intrinsic capacity of rainfall to cause erosion. Water erosion would not occur if all rain were non - erosive, therefore erosivity is fond of the physical properties of rainfall. Rainfall indices represent the climatic influence of rainfall on water related soil erosion ( $\mathrm{Yu}$, 1998). The basic erosivity factors include; Amount: This is the quantity of rain that falls in any given rainfall event. The higher the amount the more erosive it becomes. Intensity: This refers to the amount of rain received during a unit time. High intensity rain are usually received during short duration, thus low intensity are of long duration. High intensity storm have large drop sizes thus will cause more erosivity. Rain-drop size: Raindrops ranges from 0.0039 inches $(0.1 \mathrm{~cm})$ to 0.35 inches $(1.0 \mathrm{~cm})$ in mean diameter above which they tend to break up. Smaller drops are called cloud droplets and their shape is spherical. As raindrop increases in size, its shape becomes more oblate with its larges cross section facing the on-coming air flow. Large raindrops are increasingly flattened on the bottom like hamburger buns and very large ones are shaped like parachutes. The large size is explained by condensation on large smoke particles or by collision between drops of liquid water. Drop size distribution: Some rain are made up of drops of all sizes, the proportion of large and small drops (size distribution) and how it varies in different rain affects erosivity. Much bigger rain drop sizes from thunder storm have high intensities, and are highly erosive as they strike the soil with combine force. Terminal velocity: Falling rain drop reach a maximum (terminal) velocity when the force of gravitational acceleration is equated by resistance to the drop falling through the air. The terminal velocity is a function of the drop size and it increases up to $9 \mathrm{~ms}^{-1}$ from the largest drop. Mathematically, terminal velocity is calculated as;

$V_{t}=\frac{2 g r^{2}(e-\sigma)}{9 N}$,

where, $\mathrm{V}_{\mathrm{t}}=$ Terminal velocity; $\mathrm{g}=$ Acceleration due to gravity; $r=$ Radius of the raindrop, $\mathrm{e}=$ Density of water, $\sigma$ $=$ Density of air $\left(1 \mathrm{kgm}^{-3}\right), \mathrm{N}=$ Viscosity of air (Assume $1 \mathrm{x}$ $\left.10^{-3}\right)$. Another factor in Erosovity determinant is the Kinetic Energy of Rainfall. This is the force impact of rain and it is a major factor in splash erosion. It is mathematically calculated as; $K E=210.3+89 \log _{10} I$,

where, $\mathrm{KE}=$ Kinetic energy $\left(\right.$ metric ton $\left.\mathrm{ha}^{-1}\right) ; \mathrm{I}=$ Rainfall intensity $\left(\mathrm{mmhr}^{-1}\right)$. 


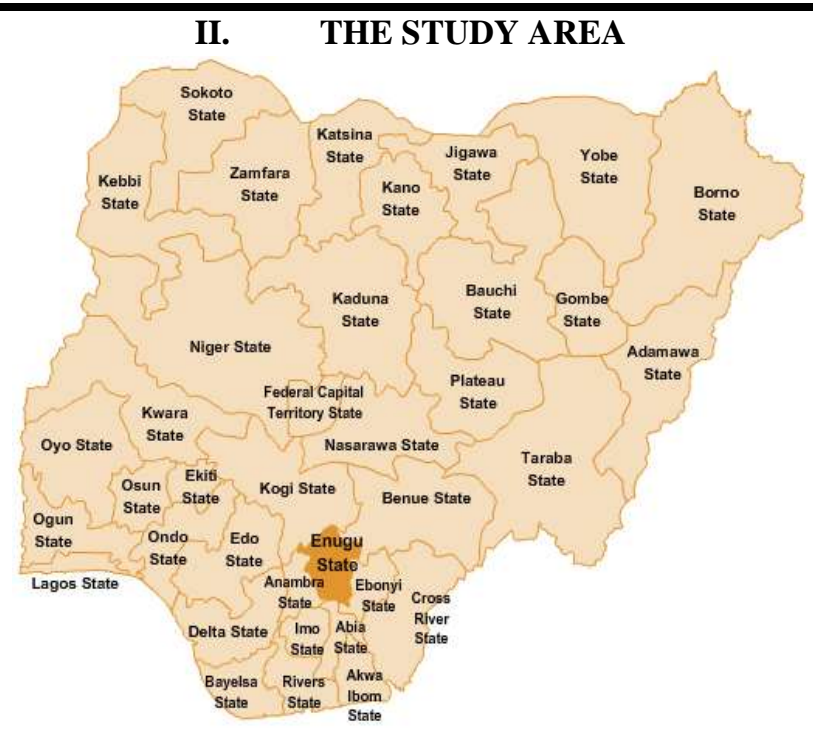

The study area Enugu State of Nigeria in West Africa is bounded by several other states; in the North by Benue and Kogi states, in the South by Abia and Imo states, while in the West and East by Anambra and Ebonyi State respectively. Minerals mined in Enugu state includes; coal, iron ore, fine clay, silica sand, lime stone, and marble (Wikipedia, 2014). The climate is tropical hinterland in nature and is comparatively congenial, characterized by high temperature, high humidity and substantial rainfall which is entirely seasoned, most of it falling between May and October. Rainfalls in the tropical regions are more erosive than those in the temperate regions due to the presence of strong winds and high temperature. Annual distributions of rainfall also influence the erosivity of rain.

\section{MATERIALS}

The major materials used in this work are the rainguage, the automated scientific weather station, and rainfall data. Rainfall data include rainfall amounts and duration. These rainfall records were obtained using the rain gauge and the automated scientific weather station which is equipped with a standard set of sensors that record; air temperature, relative humidity, rainfall amount, duration, speed and direction, soil temperature and moisture, etc., automatically every five minutes interval.

\section{METHODOLOGY}

Rainfall amount, A (mm) was obtained by daily reading of the rainfall while rainfall duration, $\mathrm{T}$ (hr) was obtained by timing each rainstorm using the scientific automated weather station. These rainfall records were carried out in the four geographical zones of the study area; Enugu North (Nsukka), Enugu South (Awgu), Enugu West (Udi), and
Enugu East (Enugu capital city). Erosivity indices were calculated as below;

\section{i. The Peak storm intensity index (AIM):}

This erosivity index is the product of the amount of rain per storm and the peak intensity of storm. This index was developed by Lal (1976) and is generally referred to as AIM index.

The peak storm intensity index is stated as thus;

$A I M=A(m m) x I M\left(m m h r^{-1}\right) \quad-$

Where, $\mathrm{A}=$ Total Amount of rainfall; $\mathrm{IM}=$ Maximum rainfall intensity (peak intensity) within the period.

Procedures: Identify the total rainfall amount, A (total in month) from the daily rainfall data (Appendix 4) and multiply it with the maximum rainfall intensity that occurred in that month. That is; A (mm) x IM $\left(\mathrm{mmhr}^{-1}\right)$. Multiply the result by $\left(10^{-2}\right)$ to convert the unit $\mathrm{mm}^{2} \mathrm{hr}^{-1}$ to $\mathrm{cm}^{2} \mathrm{hr}^{-1}$. Example: In January 2014, Total amount, A = $58.00 \mathrm{~mm}$, and maximum Intensity, I occurred on $27^{\text {th }}$ day with $229.17 \mathrm{mmhr}^{-1}$ (From appendix 2.1.1). Thus;

Jan. $2014=58.00 \mathrm{~mm} \mathrm{X} 229.17 \mathrm{mmhr}^{-1}=13291.86 \mathrm{~mm}^{2} \mathrm{hr}^{-1} \mathrm{x}$ $10^{-2}=132.9186 \mathrm{~cm}^{2} \mathrm{hr}^{-1}$; or Jan. $2014=58.00 \mathrm{~mm} \mathrm{x}$ $229.17 \mathrm{mmhr}^{-1}, \quad=5.8 \mathrm{~cm} \quad \mathrm{x} 22.917 \mathrm{cmhr}^{-1},=$ $132.9186 \mathrm{~cm}^{2} \mathrm{hr}^{-1}$

\section{ii. The Kinetic Energy of Rainfall (KE):}

Kinetic energy of rainfall is the force impact of rainfall, its role in soil detachment has long been recognized (Ellison, 1944), It is mathematically defined using the energy intensity relationship equation developed by Wischemeier (1969) and it stated as thus;

$K E=210.3+89 \log _{10} I \quad-\quad \quad-\quad$ (ii)

Where; KE $=$ Kinetic energy (ton metric $\mathrm{hr}^{-1}$ ); I = Rainfall intensity $\left(\mathrm{mmhr}^{-1}\right)$

iii. The Maximum 30 Minutes Intensity Index $\left(\mathbf{E I}_{30}\right)$ :

This is a product of kinetic energy of storm and the 30minutes intensity (greatest intensity during any 30 minute period). $\mathrm{EI}_{30}$ Index was computed from rainfall data by locating the greatest amount of rain that fell in any 30 minutes intensity, and multiplying the value with Kinetic energy. Wischemeier (1969) found this index to be most significantly suitable for erosion.

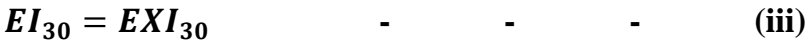

Where; $\mathrm{E}=$ kinetic energy of rainfall; $\mathrm{I}_{30}=$ Maximum 30minutes intensity

\section{iv. The Kinetic Energy Greater Than 25 Index (KE >} 25)

This is the force impact of rain that is capable of causing erosion. Thus, this index is used to separate erosive rain from non erosive rain (KE $>25$ index is used to identify

Page | 73 
erosive rain). Hudson (1971) developed KE > 25 index after he found accumulated Kinetic energy of storms with intensity greater than $25 \mathrm{mmhr}^{-1}$ with soil loss. This method was based upon the concept that there is a threshold value of intensity at which it starts to be erosive. This value is $25 \mathrm{mmhr}^{-1}$. Mathematically;
$\boldsymbol{K} \boldsymbol{E}>25=210.3+89 \log _{\mathbf{1 0}}(\boldsymbol{I}>25) \quad-\quad$ -

(iv)

Where; I $>25=$ Intensities above $25 \mathrm{mmhr}^{-1} ; \mathrm{I}=$ Rainfall intensity $\left(\mathrm{mmhr}^{-1}\right)$.

\section{RESULTS AND DISCUSSION}

The following results were generated using the procedures discussed above. The procedure was generated for other months and years and was tabulated as thus;

Table.1: Peak Storm Intensity Index (AIM) for year 2014.

\begin{tabular}{|l|c|c|c|}
\hline Month & $\begin{array}{l}\text { TOTAL RAINFALL } \\
\text { AMOUNT, A (mm) }\end{array}$ & $\begin{array}{l}\text { MAXIMUM RAINFALL } \\
\left.\text { INTENSITY, IM (mmhr }{ }^{-1}\right)\end{array}$ & $\begin{array}{l}\text { PEAK STORM INTENSITY } \\
\text { INDEX, AIM }\left(\mathrm{cm}^{2} \mathrm{hr}^{-1}\right) \\
\text { A IM IM }\end{array}$ \\
\hline Jan & 58.00 & 229.17 & 132.9186 \\
\hline Feb & 8.00 & 72.73 & 113.9506 \\
\hline March & 78.50 & 145.16 & 257.4000 \\
\hline April & 156.00 & 165.00 & 236.0966 \\
\hline May & 169.50 & 139.29 & 810.5387 \\
\hline June & 371.50 & 218.18 & 181.5100 \\
\hline July & 214.50 & 84.62 & 388.6831 \\
\hline Aug & 211.00 & 184.21 & 714.375 \\
\hline Sept & 381.00 & 187.50 & 68.6511 \\
\hline Oct & 59.50 & 115.38 & 134.7517 \\
\hline Nov & 60.50 & 222.73 & 0.0000 \\
\hline Dec & 0.00 & 0.00 & 3044.6938 \\
\hline Total & 1768.0 & 1763.97 & \\
\hline
\end{tabular}

Note: 2014 Total rainfall Amount (A) and maximum Intensity (IM) were taken from NIMET, NWFS, Enugu State Min. Of Agriculture, while AIM index is multiplication of both A and IM.

Table.2: Peak Storm Intensity Index (AIM) for year 2013.

\begin{tabular}{|l|c|c|c|}
\hline Month & $\begin{array}{l}\text { TOTAL RAINFALL } \\
\text { AMOUNT, A }(\mathrm{mm})\end{array}$ & $\begin{array}{l}\text { MAXIMUM RAINFALL } \\
\text { INTENSITY, IM (mmhr }\end{array}$ & $\begin{array}{l}\text { PEAK STORM INTENSITY } \\
\text { INDEX, AIM }\left(\mathrm{cm}^{2} \mathrm{hr}^{-1}\right) \\
\text { A x IM }\end{array}$ \\
\hline Jan & 47 & 75.81 & 35.6307 \\
\hline Feb & 0.00 & 0.00 & 0.0000 \\
\hline March & 28.00 & 121.43 & 34.0004 \\
\hline April & 139.00 & 125.00 & 173.7500 \\
\hline May & 279.00 & 115.38 & 321.9102 \\
\hline June & 236.50 & 72.22 & 170.8003 \\
\hline July & 235.50 & 185.71 & 437.3471 \\
\hline Aug & 168.00 & 140.54 & 236.1072 \\
\hline Sept & 417.10 & 183.33 & 764.6694 \\
\hline Oct & 182.60 & 118.64 & 2042.2637 \\
\hline Nov & 59.00 & 91.89 & 54.2151 \\
\hline Dec & 2.00 & 40.00 & 0.8000 \\
\hline Total & 1793.7 & 1269.95 & 4271.4941 \\
\hline
\end{tabular}


Note: 2013 Total rainfall Amount (A) and maximum Intensity (IM) were taken from NIMET, NWFS, Enugu State Min. of Agriculture, while AIM index is multiplication of both A and IM.

Table.3: Peak Storm Intensity Index (AIM) for year 2012

\begin{tabular}{|l|c|c|c|}
\hline Month & $\begin{array}{l}\text { TOTAL RAINFALL } \\
\text { AMOUNT, A (mm) }\end{array}$ & $\begin{array}{l}\text { MAXIMUM RAINFALL } \\
\text { INTENSITY, IM }\left(\mathrm{mmhr}^{-1}\right)\end{array}$ & $\begin{array}{l}\text { PEAK STORM INTENSITY } \\
\text { INDEX, AIM }\left(\mathrm{cm}^{2} \mathrm{hr}^{-1}\right) \\
=\text { A x IM }\end{array}$ \\
\hline Jan & 4.00 & 44.44 & 1.7776 \\
\hline Feb & 3.20 & 41.67 & 1.3334 \\
\hline March & 2.80 & 26.00 & 0.7280 \\
\hline April & 139.50 & 136.36 & 190.2222 \\
\hline May & 298.00 & 157.14 & 468.2772 \\
\hline June & 269.90 & 195.45 & 527.5196 \\
\hline July & 343.50 & 175.00 & 601.1250 \\
\hline Aug & 161.00 & 133.33 & 214.6613 \\
\hline Sept & 223.00 & 142.31 & 317.3513 \\
\hline Oct & 265.00 & 172.22 & 61.2500 \\
\hline Nov & 49.00 & 125.00 & 0.0000 \\
\hline Dec & 0.00 & 0.00 & 2840.6286 \\
\hline Total & 1758.9 & 1348.92 & . \\
\hline
\end{tabular}

Note: 2012 Total rainfall Amount (A) and maximum Intensity (IM) were taken from NIMET, NWFS, Enugu State Min. of Agriculture, while AIM index is multiplication of both A and IM.

Table.4: Peak Storm Intensity Index (AIM) for year 2011.

\begin{tabular}{|c|c|c|c|}
\hline Month & $\begin{array}{l}\text { TOTAL RAINFALL } \\
\text { AMOUNT, A }(\mathrm{mm})\end{array}$ & $\begin{array}{l}\text { MAXIMUM RAINFALL } \\
\text { INTENSITY, IM }\left(\mathrm{mmhr}^{-1}\right)\end{array}$ & $\begin{array}{l}\text { PEAK STORM INTENSITY } \\
\text { INDEX, AIM }\left(\mathrm{cm}^{2} \mathrm{hr}^{-1}\right) \\
=\mathrm{A} \times \mathrm{IM}\end{array}$ \\
\hline Jan & 0.00 & 0.00 & 0.0000 \\
\hline Feb & 112.00 & 52.24 & 58.5088 \\
\hline March & 96.60 & 68.00 & 65.6880 \\
\hline April & 223.40 & 71.96 & 160.7586 \\
\hline May & 454.80 & 164.55 & 748.3734 \\
\hline June & 637.60 & 162.00 & 1032.9120 \\
\hline July & 482.40 & 174.00 & 839.3760 \\
\hline Aug & 281.60 & 153.85 & 433.2416 \\
\hline Sept & 581.60 & 161.54 & 939.5166 \\
\hline Oct & 175.50 & 111.11 & 194.9981 \\
\hline Nov & 0.00 & 0.00 & 0.0000 \\
\hline Dec & 0.00 & 0.00 & 0.0000 \\
\hline Total & 3045.5 & 1119.25 & 4473.3731 \\
\hline
\end{tabular}

Note: 2011 Total rainfall Amount (A) and maximum Intensity (IM) were taken from NIMET, NWFS, Enugu State Min. of Agriculture, while AIM index is multiplication of both A and IM.

Table.5: Peak Storm Intensity Index (AIM) for year 2010.

\begin{tabular}{|l|c|c|c|}
\hline Month & $\begin{array}{l}\text { TOTAL RAINFALL } \\
\text { AMOUNT, A }(\mathrm{mm})\end{array}$ & $\begin{array}{l}\text { MAXIMUM RAINFALL } \\
\text { INTENSITY, IM }\left(\mathrm{mmhr}^{-1}\right)\end{array}$ & $\begin{array}{l}\text { PEAK STORM INTENSITY } \\
\text { INDEX, AIM }\left(\mathrm{cm}^{2} \mathrm{hr}^{-1}\right) \\
=\mathrm{A} \times \mathrm{IM}\end{array}$ \\
\hline Jan & 16.60 & 70.91 & 11.7711 \\
\hline Feb & 16.00 & 136.00 & 21.7600 \\
\hline March & 14.40 & 160.00 & 23.0400 \\
\hline April & 448.60 & 174.29 & 781.8649 \\
\hline
\end{tabular}




\begin{tabular}{|l|c|c|c|}
\hline May & 354.40 & 166.67 & 590.6785 \\
\hline June & 658.00 & 142.86 & 940.0188 \\
\hline July & 808.20 & 157.14 & 1270.0055 \\
\hline Aug & 734.40 & 197.27 & 1448.7509 \\
\hline Sept & 852.60 & 152.00 & 1295.9520 \\
\hline Oct & 456.20 & 148.00 & 675.1760 \\
\hline Nov & 0.00 & 0.00 & 0.0000 \\
\hline Dec & 0.00 & 0.00 & 0.0000 \\
\hline Total & 4359.4 & 1505.14 & 6118.9989 \\
\hline
\end{tabular}

Note: 2010 Total rainfall Amount (A) and maximum Intensity (IM) were taken from NIMET, NWFS, Enugu State Min. of Agriculture, while AIM index is multiplication of both A and IM.

Table.6: Peak Storm Intensity Index (AIM) for year 2009.

\begin{tabular}{|l|c|c|c|}
\hline Month & $\begin{array}{l}\text { TOTAL RAINFALL } \\
\text { AMOUNT, A (mm) }\end{array}$ & $\begin{array}{l}\text { MAXIMUM RAINFALL } \\
\text { INTENSITY, IM }\left(\mathrm{mmhr}^{-1}\right)\end{array}$ & $\begin{array}{l}\text { PEAK STORM INTENSITY } \\
\text { INDEX, AIM }\left(\mathrm{cm}^{2} \mathrm{hr}^{-1}\right) \\
\text { A x IM }\end{array}$ \\
\hline Jan & 66.00 & 131.91 & 87.0606 \\
\hline Feb & 0.60 & 30.00 & 0.1800 \\
\hline March & 36.40 & 173.33 & 63.0921 \\
\hline April & 378.40 & 160.00 & 605.4400 \\
\hline May & 776.00 & 166.67 & 1293.3592 \\
\hline June & 432.20 & 176.92 & 764.6482 \\
\hline July & 736.60 & 165.52 & 7219.2203 \\
\hline Aug & 506.80 & 157.11 & 922.7239 \\
\hline Sept & 541.60 & 170.37 & 1553.2020 \\
\hline Oct & 756.00 & 205.45 & 147.0445 \\
\hline Nov & 106.20 & 138.46 & 0.0000 \\
\hline Dec & 0.00 & 0.00 & 7452.2043 \\
\hline Total & 4336.8 & 1675.74 & . \\
\hline
\end{tabular}

Note: 2009 Total rainfall Amount (A) and maximum Intensity (IM) were taken from NIMET, NWFS, Enugu State Min. of Agriculture, while AIM index is multiplication of both A and IM.

Table.7: Kinetic Energy of Rainfall for year 2014.

\begin{tabular}{|l|c|c|}
\hline Month & $\begin{array}{l}\text { RAINFALL INTENSITY, I } \\
\left(\mathrm{mmhr}^{-1}\right)\end{array}$ & $\begin{array}{l}\text { KINETIC ENERGY, KE }\left(\mathrm{metric}^{2} \text { ton } \mathrm{ha}^{-1}\right)= \\
210.3+89 \log _{10}(\mathrm{I})\end{array}$ \\
\hline Jan & 304.17 & $431.2959 \times 10^{-6}$ \\
\hline Feb & 72.73 & $375.9913 \times 10^{-6}$ \\
\hline March & 402.74 & $442.1450 \times 10^{-6}$ \\
\hline April & 550.01 & $454.1956 \times 10^{-6}$ \\
\hline May & 601.34 & $457.6399 \times 10^{-6}$ \\
\hline June & 1030.23 & $478.4481 \times 10^{-6}$ \\
\hline July & 481.38 & $449.0425 \times 10^{-6}$ \\
\hline Aug & 640.01 & $460.0518 \times 10^{-6}$ \\
\hline Sept & 1028.87 & $478.4036 \times 10^{-6}$ \\
\hline Oct & 277.63 & $427.7715 \times 10^{-6}$ \\
\hline Nov & 372.73 & $439.1546 \times 10^{-6}$ \\
\hline Dec & 0.00 & $210.3000 \times 10^{-6}$ \\
\hline Total & 5761.84 & $4673.1439 \times 10^{-6}$ \\
\hline
\end{tabular}

Note: 2014 rainfall intensity, I $\left(\mathrm{mmhr}^{-1}\right)$ were taken from NIMET, NWFS, Enugu State Min. of Agriculture. 
Table.8: Kinetic Energy of Rainfall for year 2013.

\begin{tabular}{|l|c|c|}
\hline Month & $\begin{array}{l}\text { RAINFALL INTENSITY, I } \\
\left(\mathrm{mmhr}^{-1}\right)\end{array}$ & $\begin{array}{l}\text { KINETIC ENERGY, KE }\left(\mathrm{metric}^{2} \text { ton } \mathrm{ha}^{-1}\right)= \\
210.3+89 \log _{10}(\mathrm{I})\end{array}$ \\
\hline Jan & 75.81 & $377.5933 \times 10^{-6}$ \\
\hline Feb & 0.00 & $210.3000 \times 10^{-6}$ \\
\hline March & 206.05 & $416.2460 \times 10^{-6}$ \\
\hline April & 563.75 & $455.1479 \times 10^{-6}$ \\
\hline May & 751.03 & $466.2373 \times 10^{-6}$ \\
\hline June & 361.12 & $437.9353 \times 10^{-6}$ \\
\hline July & 751.68 & $466.2640 \times 10^{-6}$ \\
\hline Aug & 428.67 & $444.5569 \times 10^{-6}$ \\
\hline Sept & 1332 & $488.3805 \times 10^{-6}$ \\
\hline Oct & 536.63 & $453.2433 \times 10^{-6}$ \\
\hline Nov & 181.18 & $411.2709 \times 10^{-6}$ \\
\hline Dec & 40 & $352.8869 \times 10^{-6}$ \\
\hline Total & 5227.92 & $4980.0623 \times 10^{-6}$ \\
\hline
\end{tabular}

Note: 2013 rainfall intensity, I $\left(\mathrm{mmhr}^{-1}\right)$ were taken from NIMET, NWFS, Enugu State Min. of Agriculture.

Table.9: Kinetic Energy of Rainfall for year 2012.

\begin{tabular}{|l|c|c|}
\hline Month & $\begin{array}{l}\text { RAINFALL INTENSITY, I } \\
\left(\mathrm{mmhr}^{-1}\right)\end{array}$ & $\begin{array}{l}\text { KINETIC ENERGY, KE }\left(\mathrm{metric}^{2} \text { ton } \mathrm{ha}^{-1}\right)= \\
210.3+89 \log _{10}(\mathrm{I})\end{array}$ \\
\hline Jan & 44.44 & $356.9542 \times 10^{-6}$ \\
\hline Feb & 65.00 & $371.6481 \times 10^{-6}$ \\
\hline March & 44.75 & $357.2212 \times 10^{-6}$ \\
\hline April & 719.66 & $464.5819 \times 10^{-6}$ \\
\hline May & 1343.88 & $488.7276 \times 10^{-6}$ \\
\hline June & 1350.25 & $488.9056 \times 10^{-6}$ \\
\hline July & 1584.1 & $495.0822 \times 10^{-6}$ \\
\hline Aug & 788.16 & $468.0974 \times 10^{-6}$ \\
\hline Sept & 133.13 & $488.3271 \times 10^{-6}$ \\
\hline Oct & 1631.14 & $496.3905 \times 10^{-6}$ \\
\hline Nov & 206.82 & $416.3884 \times 10^{-6}$ \\
\hline Dec & 0.00 & $210.3000 \times 10^{-6}$ \\
\hline Total & 7911.33 & $5102.6242 \times 10^{-6}$ \\
\hline
\end{tabular}

Note: 2012 rainfall intensity, I $\left(\mathrm{mmhr}^{-1}\right)$ were taken from NIMET, NWFS, Enugu State Min. of Agriculture.

Table.10: Kinetic Energy of Rainfall for year 2011.

\begin{tabular}{|l|c|c|}
\hline Month & $\begin{array}{l}\text { RAINFALL INTENSITY, I } \\
\left(\mathrm{mmhr}^{-1}\right)\end{array}$ & $\begin{array}{l}\text { KINETIC ENERGY, KE }\left(\mathrm{metric}^{2} \text { ton } \mathrm{ha}^{-1}\right)= \\
210.3+89 \log _{10}(\mathrm{I})\end{array}$ \\
\hline Jan & 0.00 & $210.3000 \times 10^{-6}$ \\
\hline Feb & 145.45 & $402.7803 \times 10^{-6}$ \\
\hline March & 129.28 & $398.2235 \times 10^{-6}$ \\
\hline April & 245.48 & $423.0100 \times 10^{-6}$ \\
\hline May & 1059.71 & $479.5428 \times 10^{-6}$ \\
\hline June & 1298.59 & $487.4015 \times 10^{-6}$ \\
\hline July & 880.77 & $472.3961 \times 10^{-6}$ \\
\hline Aug & 857.56 & $471.3637 \times 10^{-6}$ \\
\hline Sept & 1637.42 & $496.3638 \times 10^{-6}$ \\
\hline Oct & 422.19 & $443.9695 \times 10^{-6}$ \\
\hline
\end{tabular}




\begin{tabular}{|l|c|c|}
\hline Nov & 0.00 & $210.3000 \times 10^{-6}$ \\
\hline Dec & 0.00 & $210.3000 \times 10^{-6}$ \\
\hline Total & 6676.45 & $4714.9512 \times 10^{-6}$ \\
\hline
\end{tabular}

Note: 2011 rainfall intensity, I $\left(\mathrm{mmhr}^{-1}\right)$ were taken from NIMET, NWFS, Enugu State Min. of Agriculture.

Table.11: Kinetic Energy of Rainfall for year 2010.

\begin{tabular}{|l|c|c|}
\hline Month & $\begin{array}{l}\text { RAINFALL INTENSITY, I } \\
\left(\mathrm{mmhr}^{-1}\right)\end{array}$ & $\begin{array}{l}\text { KINETIC ENERGY, KE }\left(\mathrm{metric}^{2} \text { ton } \mathrm{ha}^{-1}\right)= \\
210.3+89 \log _{10}(\mathrm{I})\end{array}$ \\
\hline Jan & 104.24 & $389.9020 \times 10^{-6}$ \\
\hline Feb & 184.00 & $411.8672 \times 10^{-6}$ \\
\hline March & 160.00 & $406.4649 \times 10^{-6}$ \\
\hline April & 930.12 & $474.4965 \times 10^{-6}$ \\
\hline May & 904.66 & $473.4285 \times 10^{-6}$ \\
\hline June & 950.59 & $475.3420 \times 10^{-6}$ \\
\hline July & 1353.60 & $489.0035 \times 10^{-6}$ \\
\hline Aug & 1594.00 & $495.3225 \times 10^{-6}$ \\
\hline Sept & 1504.43 & $493.0886 \times 10^{-6}$ \\
\hline Oct & 826.17 & $469.9219 \times 10^{-6}$ \\
\hline Nov & 0.00 & $210.3000 \times 10^{-6}$ \\
\hline Dec & 0.00 & $210.3000 \times 10^{-6}$ \\
\hline Total & 8511.81 & $4999.4376 \times 10^{-6}$ \\
\hline
\end{tabular}

Note: 2010 rainfall intensity, I $\left(\mathrm{mmhr}^{-1}\right)$ were taken from NIMET, NWFS, Enugu State Min. of Agriculture.

Table.12: Kinetic Energy of Rainfall for year 2009.

\begin{tabular}{|l|c|c|}
\hline Month & $\begin{array}{l}\text { RAINFALL INTENSITY, I } \\
\left(\mathrm{mmhr}^{-1}\right)\end{array}$ & $\begin{array}{l}\text { KINETIC ENERGY, KE }\left(\mathrm{metric}^{-1} \text { ton } \mathrm{ha}^{-1}\right)= \\
210.3+89 \log _{10}(\mathrm{I})\end{array}$ \\
\hline Jan & 189.05 & $412.9174 \times 10^{-6}$ \\
\hline Feb & 30.00 & $341.7619 \times 10^{-6}$ \\
\hline March & 173.33 & $409.5621 \times 10^{-6}$ \\
\hline April & 842.98 & $470.6962 \times 10^{-6}$ \\
\hline May & 1087.32 & $480.5396 \times 10^{-6}$ \\
\hline June & 843.42 & $470.7140 \times 10^{-6}$ \\
\hline July & 1376.43 & $489.6532 \times 10^{-6}$ \\
\hline Aug & 1294.48 & $487.2769 \times 10^{-6}$ \\
\hline Sept & 1257.37 & $486.1555 \times 10^{-6}$ \\
\hline Oct & 1161.64 & $483.0939 \times 10^{-6}$ \\
\hline Nov & 245.39 & $423.0011 \times 10^{-6}$ \\
\hline Dec & 0.00 & $210.3000 \times 10^{-6}$ \\
\hline Total & 8501.41 & $5165.6718 \times 10^{-6}$ \\
\hline
\end{tabular}

Note: 2009 rainfall intensity, I $\left(\mathrm{mmhr}^{-1}\right)$ were taken from NIMET, NWFS, Enugu State Min. of Agriculture.

Table.13: The Maximum 30 Minutes Intensity Index (EI $\left.I_{30}\right)$.

\begin{tabular}{|c|c|c|c|}
\hline YEAR & $\begin{array}{l}\text { KINETIC ENERGY, E } \\
\left(\mathrm{metric} \mathrm{ton} \mathrm{ha}^{-1}\right)\end{array}$ & $\begin{array}{l}\text { MAXIMUM 30MINUTES } \\
\text { INTENSITY, } \begin{array}{l}\mathrm{I}_{30}\left(\mathrm{mmhr}^{-1}\right)\end{array}\end{array}$ & $\begin{array}{l}\text { MAXIMUM 30MINUTES INTENSITY } \\
\text { INDEX, EI }_{30}=\mathrm{E} \mathrm{X} \mathrm{I} \mathrm{I0}_{30}\left(\mathrm{mmhr}^{-1}\right)\end{array}$ \\
\hline 2014 & $4673.1439 \times 10^{-6}$ & 139.29 & $650922.2138 \times 10^{-6}$ \\
\hline 2013 & $4980.0623 \times 10^{-6}$ & 42.55 & $211901.6509 \times 10^{-6}$ \\
\hline 2012 & $5102.6242 \times 10^{-6}$ & 42.55 & $217116.6597 \times 10^{-6}$ \\
\hline 2011 & $4714.9512 \times 10^{-6}$ & 123.27 & $581212.0344 \times 10^{-6}$ \\
\hline 2010 & $4999.4376 \times 10^{-6}$ & 139.17 & $695771.7308 \times 10^{-6}$ \\
\hline 2009 & $5165.6718 \times 10^{-6}$ & 133.33 & $688739.0211 \times 10^{-6}$ \\
\hline Average & $4939.3152 \times 10^{-6}$ & 103.36 & $507610.5518 \times 10^{-6}$ \\
\hline
\end{tabular}


Table.14: Yearly Kinetic Energy, Kinetic Energy Greater Than 25, Peak Storm Intensity, and Erosivity.

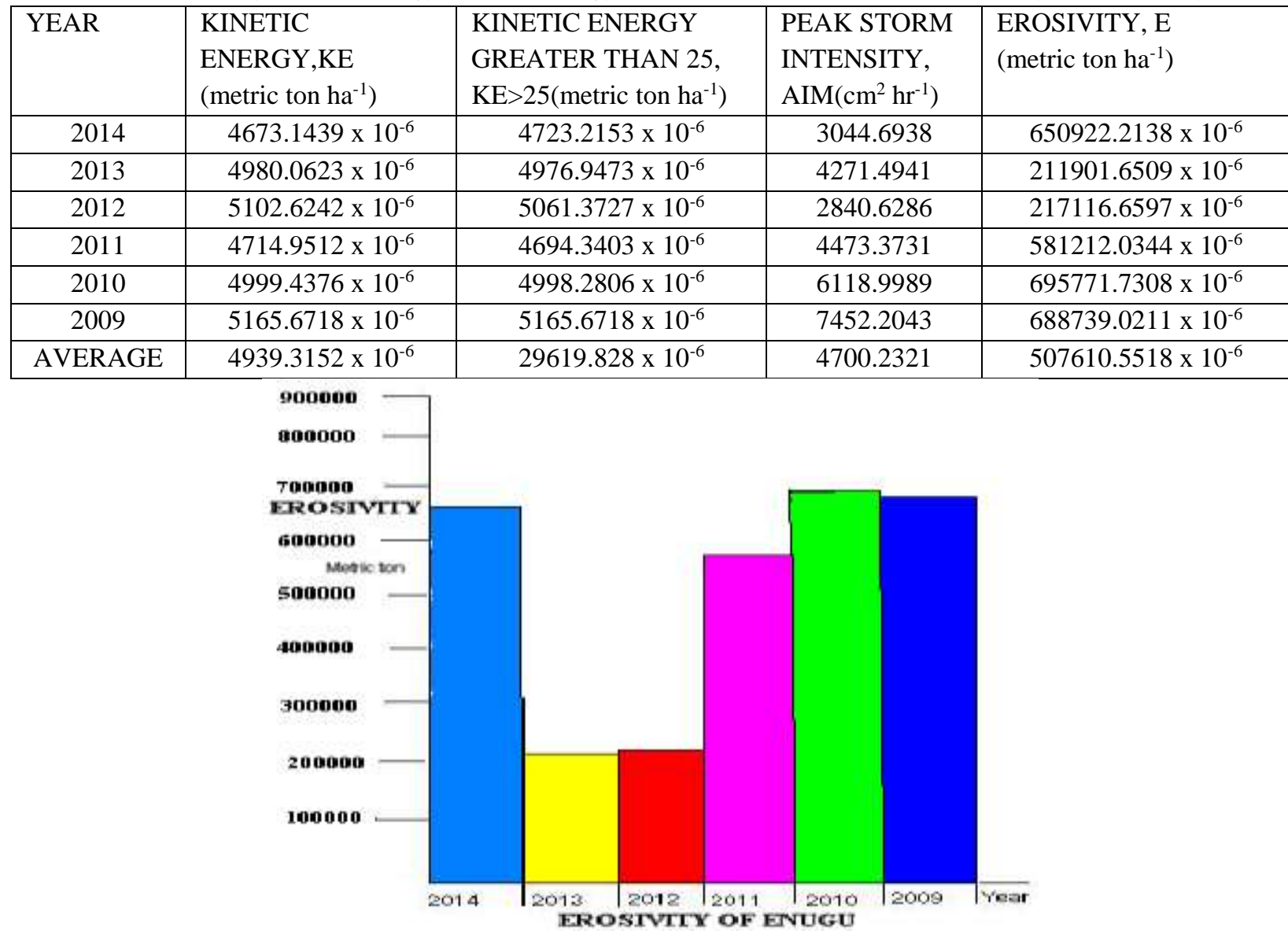

Fig. 1: Erosivity of Enugu.
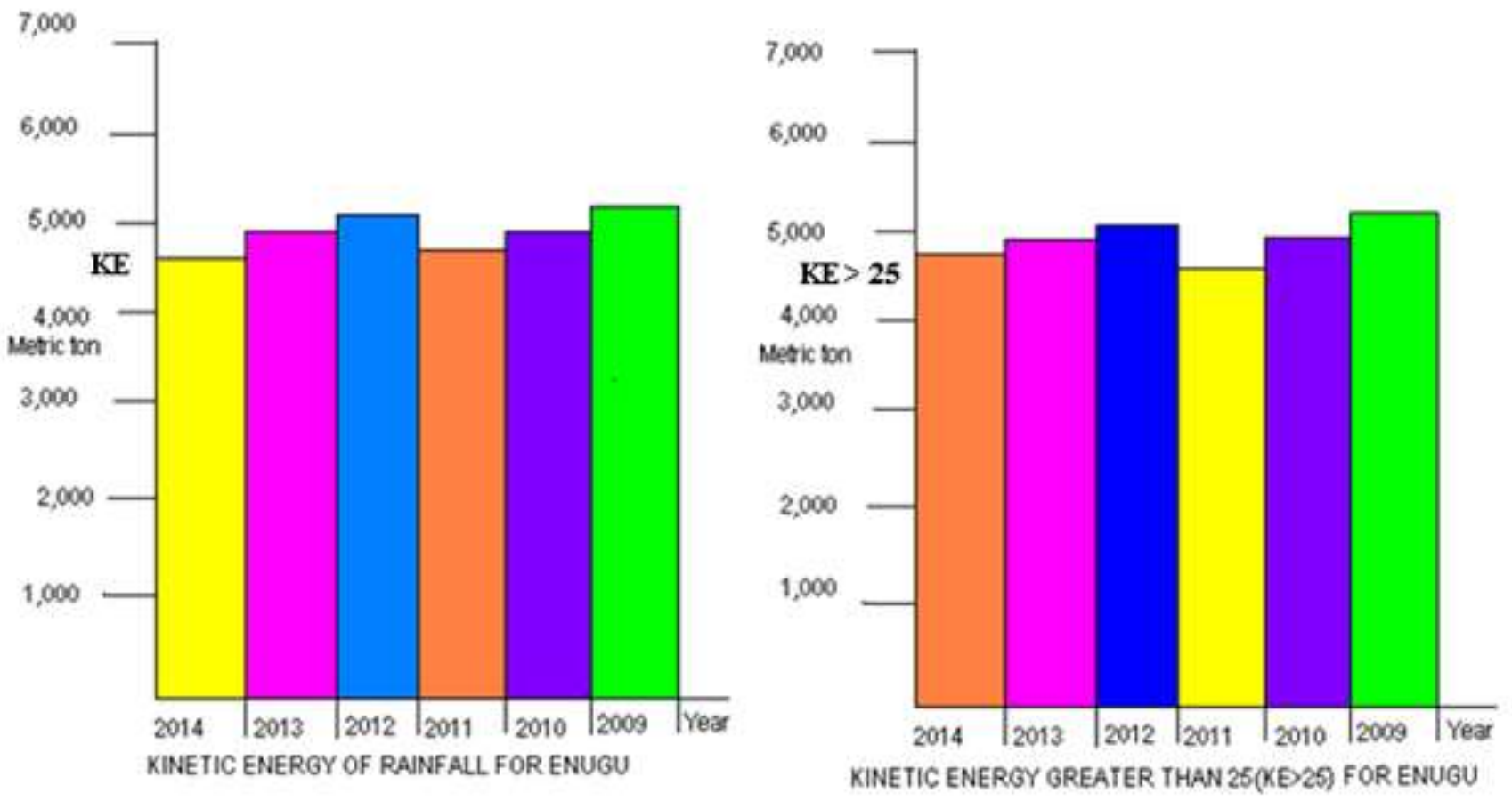

Fig. 2: Kinetic Energy and Kinetic Energy Greater Than 25 index Chart for Enugu Region. 

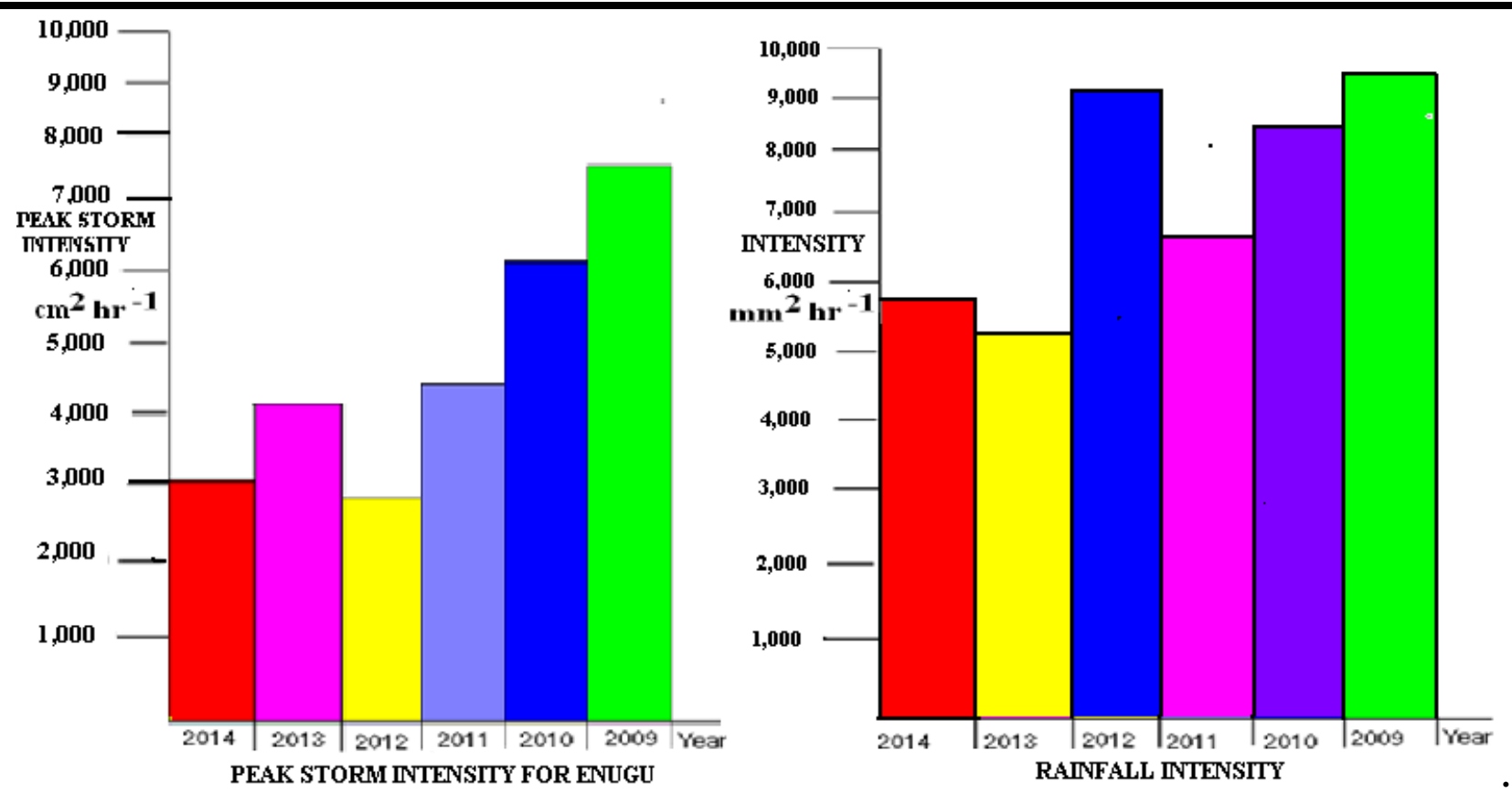

Fig.3: Peak Storm Intensity and Rainfall Intensity Chart for Enugu Region.
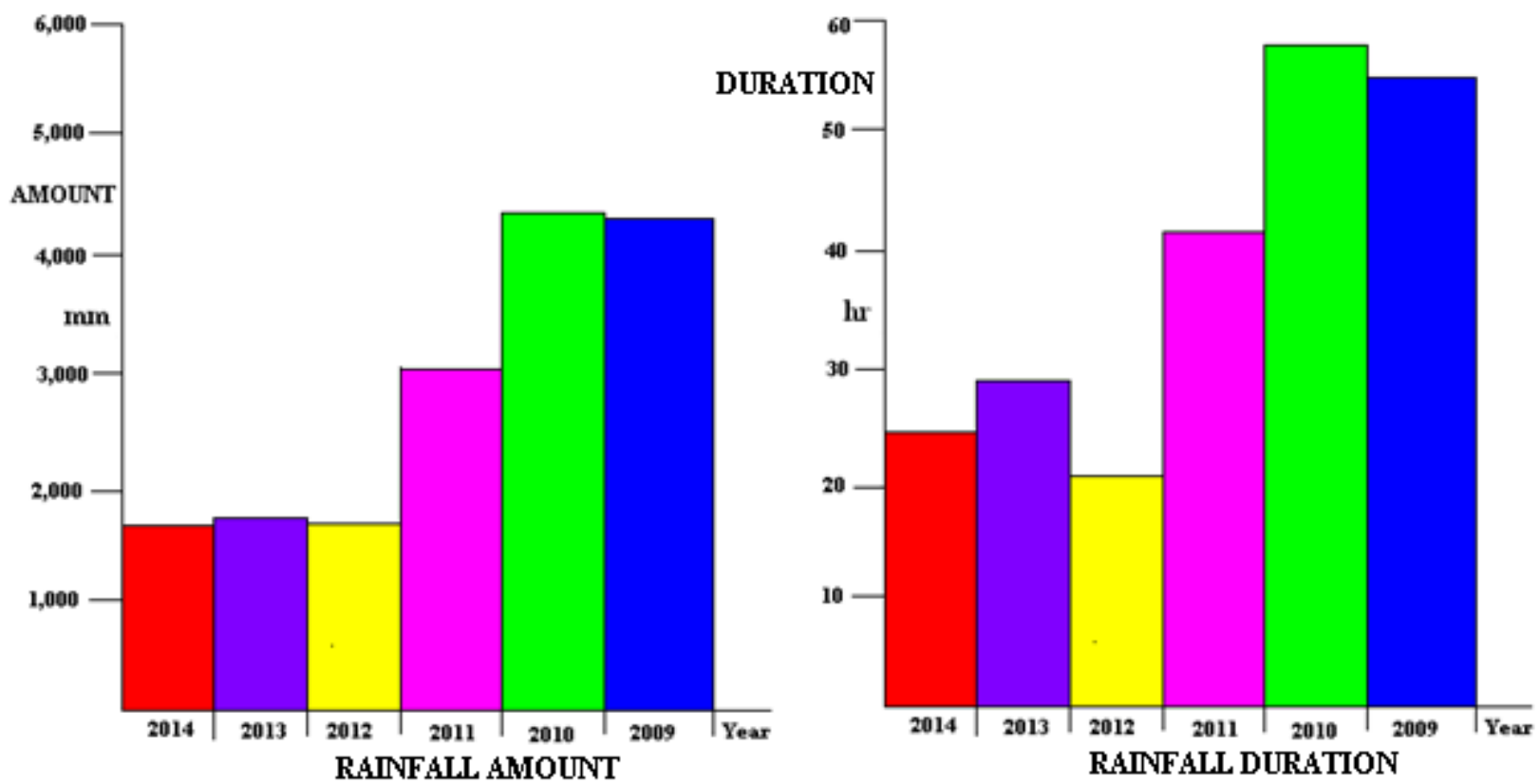

Fig. 4: Rainfall Amount and Rainfall Duration of Enugu.

\section{DISCUSSIONS}

From the above calculations, Erosivity (R) in Enugu area is $507610.5518 \times 10^{-6}$. This is equivalent to 0.5076105518 and by approximation, it is 0.5 . From the results, Erosivity (R) in Enugu area is not very intense since the value is $507610.5518 \times 10^{-6}$ or 0.51 .Erosive rains were separated from non erosive rains in tables 1 to 6 . This method maybe deployed in forecasting future erosive rains and non erosive rains. With the forecast, future erosive rainfalls, preventive www.ijeab.com measures may be carried out in spotted locations prior predictions. From universal soil loss equation, annual erosion equals the product of all the erosion variables $(\mathrm{A}=$ RKLSCP), thus if Erosivity, $\mathrm{K}$ is well manage to minimal, erosion will not occur in the controlled locations.

\section{CONCLUSION}

The knowledge of Erosivity is essential to understand erosion processes, estimate soil erosion rates, predict future 
erosive rainfall and non-erosive ones and in designing erosion control practices.

\section{RECOMMENDATION}

Conducting subsequent studies on Erosivity in the future years will help monitor any change.

More work and effort should be put in the prevention of erosion rather than remediation of eroded soil.

\section{REFERENCES}

[1] Atlas, 2016. Nigeria. World Map. Africa. Nigeria. World Atlas. pp: 1.

[2] Ellison W.D., 1944. Studies of Raindrop Erosion. Agric. Eng. 25. pp: 131.

[3] En. Wikipedia, 2014. Enugu. Article on Enugu. En.m.Wikipedia.org. pp: 1.

[4] En. Wikipedia, 2013. Enugu State Zones.svg.png, maps.pp: 27.

[5] Groisman P.Y., 1994. Rainfall equipments. Article: pp: 36.

[6] Hudson N.W., 1971. Soil Conservation, Bats-ford B.T. Bats Ford limited London. pp: 49-51.

[7] Hudson N.W., 1975. Erosion Control: Progress Report on Experiments, Henderson Research Station .Rhodesian Agric Journal. pp: 54-57.

[8] Lal R., 1976. Soil erosion problems on Alfi soil in western Nigeria and their control. IITA monograph No.1, Ibadan, Nigeria. pp: 208.

[9] Micheal and Ojah, 2005. Soil erosion and remediation. Published article on soil erosion. pp: 19.

[10] Stewart, 1975.Erosion and Soil Properties. Thesis on Erosion. pp: 28.

[11] Wischemeier W.H., 1969. Agricultural Journal on Rainfall Erosive Power of Rainfall.485;pp: 37.

[12] Wischemeier W.H and Smith D., 1978. Predicting Rainfall Erosion Losses: A Guide to Conservation Planning. USDA - ARS Agriculture Handbook No 537, Washington DC. pp: 58.

[13] Yu, 1998. Soil erosion Studies Part I. Article on Soil Erosion. pp: $19 \& 31$. 